\title{
Research on performance test analysis method of mechanical structure based on measured error / virtual simulation
}

\author{
Beilei Wang* \\ Aerospace Science \& Industry Defense Technology Research and Test Center, Beijing 100854, China
}

\begin{abstract}
Mechanical errors seriously affect the transmission performance of mechanical structure, especially for high-precision mechanical structure. With the development of virtual simulation technology, the performance test of mechanical structure in virtual environment is gradually applied. Under the background, this paper takes the movable tooth reducer as the research object and puts forward a virtual performance test method by combing test errors with virtual simulation technology. The tooth profile errors of key components of the reducer are obtained by optical scanning, and the simulation test is carried out. Meanwhile, the accuracy of the simulation test is verified by the implementation of vibration test. The results show that both the simulated vibration acceleration and the experimental test vibration acceleration show a double-peak variation trend, and the frequency difference between the two peaks is less than $5 \%$. Therefore, the simulation test method proposed in this paper can effectively evaluate the performance of mechanical structures.
\end{abstract}

Keywords: Performance test, Virtual prototype, Vibration test, Movable tooth reducer.

\section{Preface}

The performance test of mechanical structure is the key link to inspect product quality. Developing high effective and economical test methods are always of most interest for mechanical engineers. Virtual simulation technology makes this wish come true. As a new type reducer, the movable tooth reducer has broad prospects in the field of robotics and its transmission performance determines the accuracy and stability of the posture of the executive component ${ }^{[1]}$. The performance test of the reducer is a necessary technical link before industrial application ${ }^{[2]}$. The traditional method is to place the processed and assembled reducer on a test platform for performance testing. With the rise of virtual prototypes and virtual test technology ${ }^{[3]}$, a virtual performance test method by combing test errors with virtual simulation technology is performed on the reducer.

\footnotetext{
*Corresponding author's: wb17320003@163.com
} 
At present, scholars at home and abroad mainly focus on the theoretical level of the research and analysis of the performance of the reducer. The basic idea is to start with the transmission principle of the reducer, establish a theoretical calculation model, and analyze the factors affecting the performance of the reducer. Christian Habermehl ${ }^{[4]}$, et al. established a gear reducer transmission efficiency performance calculation model, calculated and analyzed the working conditions and the influence of a single component on the transmission efficiency. Chen Zhongmin ${ }^{[5]}$, et al. established the reducer system dynamics model, and solved and analyzed the vibration characteristics of the reducer. The results of this theoretical analysis have a certain deviation from the actual performance, which can be used as a guide reference basis in the design, but it cannot accurately characterize the performance parameters after actual processing. At present, the actual performance test of the reducer is to design and build a test platform, use traditional instruments to test the transmission parameters, and process the data to obtain the performance curve of the reducer ${ }^{[6]}$. It is also possible to combine the intelligent software with the test platform, integrate the data processing process into the intelligent software, and directly obtain the performance curve of the reducer ${ }^{[7]}$. At present, the existing performance test methods of reducer are finally implemented on the physical test platform of the whole machine. Although the actual performance parameters of the reducer can be obtained, the test platform is solidified, which cannot meet the performance test of multi-variety and small-batch reducers. In order to improve the flexibility of the reducer performance test, make full use of computer simulation and processing capabilities, and replace the physical test platform with a virtual prototype test platform based on actual parameters, which can well meet this technical requirement.

In this paper, takes the movable tooth reducer as the research object, and proposes a virtual performance test method based on actual parameters to predict the actual performance of the reducer. Scanner is used to obtain key component tooth profile data. Based on digital drive software, virtual prototypes with errors and virtual prototypes without errors are established. Performance tests are performed on the two sets of virtual prototypes. The vibration performance test is carried out to verify the accuracy of the virtual performance test method.

\section{Virtual performance test content of movable tooth reducer}

\subsection{Transmission efficiency}

The transmission efficiency is the ratio of the output power of the movable tooth reducer to the input power. According to the principle of mechanical transmission efficiency, the formula for calculating the efficiency of the movable tooth reducer is

$$
\eta=\frac{P_{o u t}}{P_{i n}}
$$

where: $P_{\text {in }}$ is the input power;

$P_{\text {out }}$ is the output power.

The power calculation formula is

$$
P=\frac{n T}{9550}
$$

Substituting formula (5) into formula (4), the calculation formula for virtual test transmission efficiency is 


$$
\eta=\frac{n_{\text {out }} T_{\text {out }}}{n_{\text {in }} T_{\text {in }}}
$$

where: $n_{i n}$ is the input speed; $n_{\text {out }}$ is the output speed; $T_{i n}$ is the input torque; $T_{\text {out }}$ is the output torque.

In the virtual test of its transmission efficiency, the rotation speed and torque of the input shaft and output shaft are measured. Considering that the rotation speed and torque will fluctuate in the actual test process, the test data needs to be processed and the input The input torque and output speed are averaged and their calculation formula is as follows

$$
\left\{\begin{array}{c}
\bar{n}=\frac{\sum_{i}^{N} n_{i}}{N} \\
\bar{T}=\frac{\sum_{i}^{N} T_{i}}{N}
\end{array}\right.
$$

\subsection{Vibration characteristics}

Vibration characteristics are an important performance index of the movable tooth reducer. Through virtual test and vibration response test, the vibration acceleration in the $\mathrm{X}$ direction of the output shaft is measured to obtain the actual vibration performance of the reducer.

\section{Tooth profile detection test of movable tooth prototype}

In order to accurately predict the transmission performance of the actual reducer, it is necessary to establish a virtual prototype with actual parameters. Since there is no special detection instrument for the machining error of the key components of the movable tooth reducer, a scanner is used to scan the tooth profile of the key components. The scanner used in this article is shown in Figure 1 (a). The shocker and the center wheel are the key components of the movable tooth reducer. The quality of the tooth profile processing will affect the meshing condition, and then affect the transmission performance of the reducer. Scan the tooth profile of the shocker and the center wheel to obtain the scanned point cloud image. Reverse engineering the scanned point cloud image to obtain a solid model. The model conversion is shown in Figure 1(b).

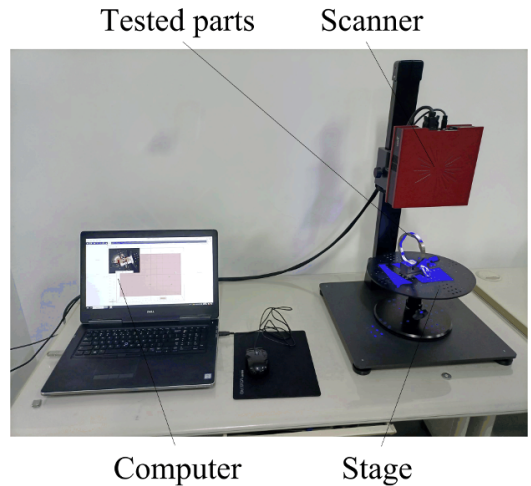

(a)

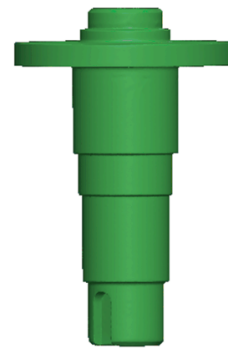

Point cloud

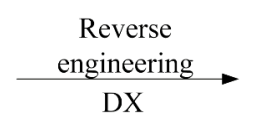

(b)

Fig. 1. Model processing. 
In order to establish a virtual prototype of the actual parameters, extract the tooth profile of the converted solid model, and store the extracted tooth profile data as a txt text file according to the coordinate value, which is used for the automatic modeling of the digital drive software. The comparison of the extracted detection tooth profile with the theoretical design tooth profile is shown in Figure 2.

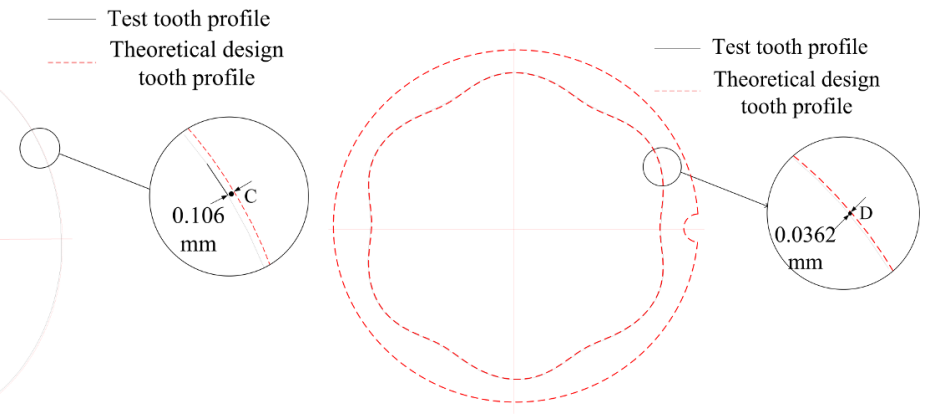

Fig. 2. Comparison of scanned tooth profile and theoretical tooth profile.

As shown in Figure 2, there is a certain tooth profile error between the detected tooth profile and the theoretically designed tooth profile. The shock profile has a deviation of $0.106 \mathrm{~mm}$ in the normal direction of point $\mathrm{C}$, and the central gear tooth profile has a deviation of $0.036 \mathrm{~mm}$ in the normal direction of point $\mathrm{D}$. In the design stage, the design accuracy of the shock is given to be $0.1 \mathrm{~mm}$, so the shock profile deviation is $0.006 \mathrm{~mm}$, and the center gear profile deviation is $0.036 \mathrm{~mm}$, indicating that the test accuracy of the scanner can reach $0.001 \mathrm{~mm}$. When the 7-level machining accuracy is adopted, the maximum deviation of the shock profile is $0.007 \mathrm{~mm}$, and the maximum deviation of the center gear profile is $0.041 \mathrm{~mm}$. The deviations of the tooth profile obtained by scanning are all within the deviation range of the theoretical profile, which further verifies the scanner's performance. The detection accuracy can meet the demand of measurement accuracy.

\section{Virtual performance test of movable gear transmission}

The error-free virtual prototype and the error virtual prototype are respectively established through the digital drive software, and the performance test and analysis of the two sets of virtual prototypes are performed using virtual testing technology.

\subsection{Virtual test of transmission efficiency}

In order to obtain the change rule of the transmission efficiency of the movable tooth reducer, the virtual test technology is used to obtain the input shaft speed and torque, and the output shaft speed and speed. The data obtained is processed by equation (4), and the transmission efficiency change can be obtained by equation (3). The rule is shown in Figure 3, the transmission efficiency of the two sets of virtual prototypes increases from 0 gradually, and then tends to level after increasing to a determined value. The transmission efficiency of the error-free virtual prototype is stable at $92 \%$, and the transmission efficiency of the virtual prototype with errors is stable at $80 \%$. The transmission efficiency of the virtual prototype with errors is reduced by $15 \%$ and tends to stabilize later, which indicates that the processing error leads to the reduction of the transmission efficiency of the movable tooth reducer. The reason is: the processing error causes the relative sliding among the movable tooth, the wave device and the center wheel to increase. Therefore, the 
force will increase, the energy consumption of the movable tooth reducer will also be greater, and the transmission efficiency will be reduced.

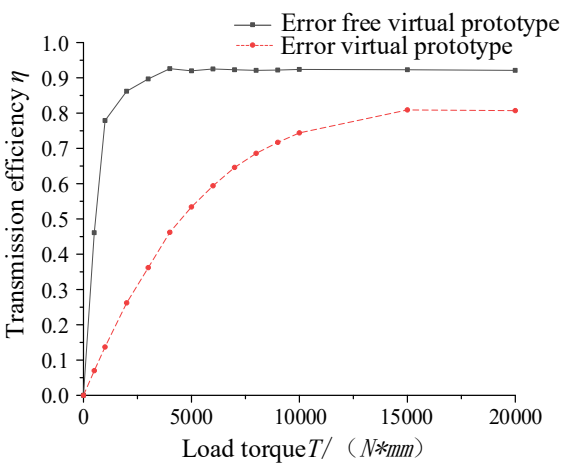

Fig. 3. The changing law of transmission efficiency.

\subsection{Virtual test of vibration characteristics}

In order to obtain the vibration characteristics of the movable tooth reducer, the frequency domain characteristics of the vibration acceleration in the $\mathrm{X}$ direction of the output shaft are obtained using virtual testing technology.

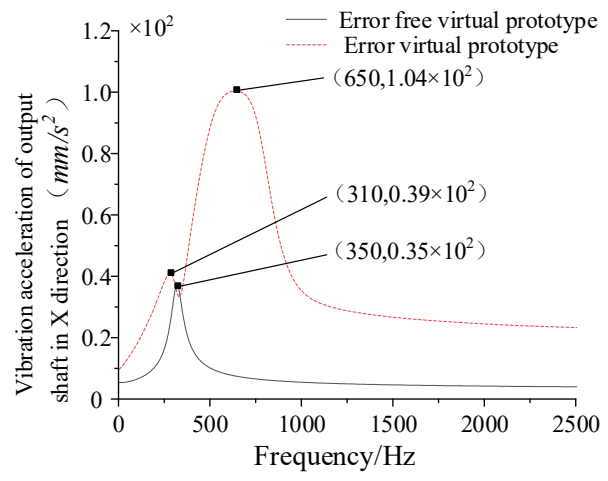

Fig. 4. Vibration frequency spectrum of the output axis in the $\mathrm{X}$ direction.

It can be seen from Figure 4 that the vibration acceleration in the $\mathrm{X}$ direction of the error-free virtual prototype has a peak value which appears at the natural frequency of the error-free virtual prototype at $350 \mathrm{~Hz}$, and the peak is $0.35 \times 102 \mathrm{~mm} / \mathrm{s}^{2}$. While the peaks in vibration accelerations in the $\mathrm{X}$ direction of the virtual prototype with errors appear at $310 \mathrm{HZ}$ and $650 \mathrm{HZ}$ respectively, which are $0.39 \times 102 \mathrm{~mm} / \mathrm{s}^{2}$ and $1.04 \times 102 \mathrm{~mm} / \mathrm{s}^{2}$. The peaks appear at the frequency multiplier of the virtual prototype with error. From the general trend, the vibration acceleration of the virtual prototype with error is greater than the error-free virtual prototype, and there are peaks at different frequencies. The reason is that the vibration acceleration of the error-free virtual prototype is mainly caused by the stiffness excitation, while the vibration acceleration of the virtual prototype with errors is caused by the stiffness excitation and the error excitation. On the one hand, the joint effect affects the vibration characteristics, on the other hand, it influences the change in frequency, which further shows that the error excitation has a greater impact on the vibration characteristics of the reducer. 


\section{Test verification}

In order to verify the accuracy of the virtual performance test, a test platform is built as shown in Figure 6. In this test, the acceleration sensor is installed at the end cover of the output shaft in the X direction. The servo motor speed is set to $1500 \mathrm{r} / \mathrm{min}$, and the load of the magnetic powder brake is set to $0.04 \mathrm{~A}\left(2000 \mathrm{~N}^{*} \mathrm{~mm}\right)$, and the sampling frequency is $5 \mathrm{KHz}$, which is consistent with the previous vibration characteristic performance test conditions.

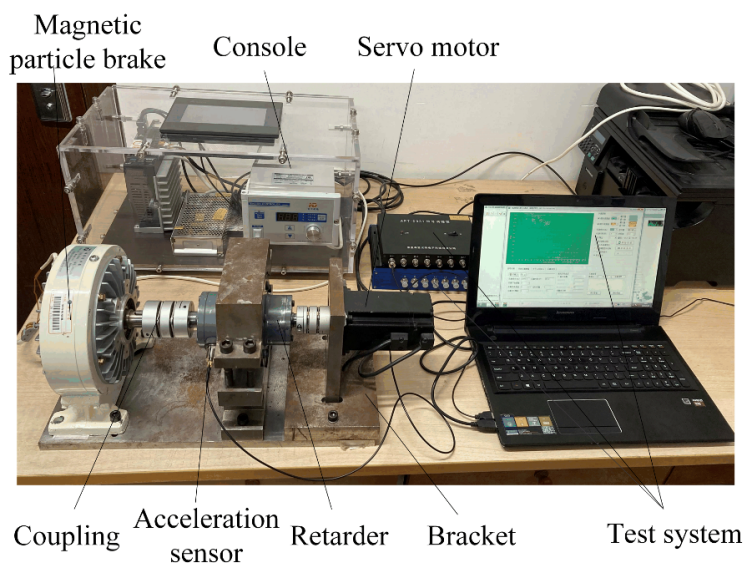

Fig. 5. Test platform.

The data acquisition software is used to perform Fourier transform on the measured test data, and the frequency spectrum of the vibration in the $\mathrm{X}$ direction of the output axis of the actual prototype is shown in Figure 6.

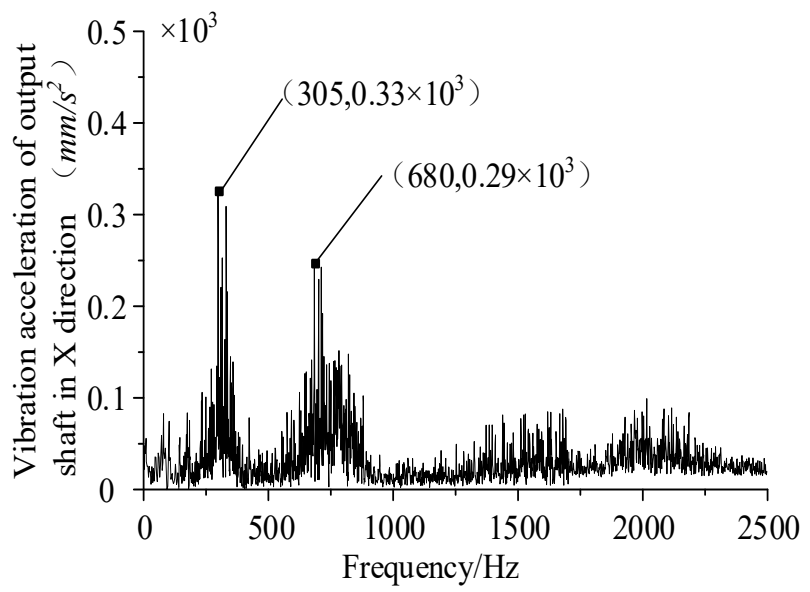

Fig. 6. Vibration frequency spectrum of the output axis in the $\mathrm{X}$ direction.

As shown in Figure 6, the frequency of the main peaks in the acceleration signal tested by the test is around $305 \mathrm{~Hz}$ and $680 \mathrm{~Hz}$, and the peak frequency of the virtual test is $310 \mathrm{~Hz}$ and $650 \mathrm{~Hz}$. The frequency phase difference rates between the two peaks are $2 \%$ and $5 \%$ which are controlled within $5 \%$. The frequency domain change law of the vibration acceleration obtained by both shows a bimodal trend, which further verifies that the virtual performance test is so accurate that it can predict the actual reducer performance to a 
certain extent. The reason why there are peaks in other positions in the test data, and there is a certain gap between the simulation and the test peaks is that in the actual test process, the influence of the assembly error of the prototype, the vibration of the test bench and environmental factors cannot be ignored, while these factors are ignored in simulation software.

\section{Conclusion}

Taking the movable tooth reducers as the research objects, the virtual performance test platform built is used to test the performances of two sets of virtual prototypes. The following conclusions are obtained combined with the vibration test to verify the accuracy of the virtual performance test method:

(1) The conclusion by comparing and analyzing the virtual performance data of the two sets of virtual prototypes is: the transmission efficiency of the virtual prototype with errors is stable at $80 \%$, the transmission efficiency of the virtual prototype with errors is reduced by $15 \%$. The transmission efficiency of the virtual prototype with errors tends to stabilize for a longer time which indicates the processing error causes the transmission efficiency of the movable gear reducer to decrease.

(2) Comparing and analyzing the frequency domain data of the vibration characteristics of the two sets of virtual prototypes, it is obvious that the processing error causes the vibration acceleration amplitude to increase significantly and the frequency at the peak to change. It can be indicated that the error excitation has a greater impact on the vibration characteristics of the reducer.

(3) The conclusion by comparing and analyzing virtual performance test data and experimental test data is: the vibration acceleration frequency domain responses of the two have the same changing trend, and the difference rates of the vibration acceleration frequency domain response are within $5 \%$, which verifies the accuracy of the virtual performance test method.

\section{References}

1. Qiao Xuetao, Zhang Libin, Yan Cunfu, et al.(2019) Analysis of the development status of my country's industrial robot RV reducer. Mechanical Strength, 41(06), 1486-1492.

2. Shi Zhaoyao, Xu Hang, Han Fangxu, et al.(2018) Current status and trend of precision reducer hysteresis measurement. Optics and Precision Engineering, 26(09), 2150-2158.

3. LI Bin, XU Shaoshuai, ZHANG Hong, et al. Research on the virtual test technique of tower crane jib. Applied Mechanics and Materials, 2012, 2025:521-525.

4. Christian Habermehl, Georg Jacobs, Stephan Neumann.(2020) A modeling method for gear transmission efficiency in transient operating conditions. Mechanism and Machine Theory, 2020, 153.

5. CHEN Zhongmin, QU Yue, LONG Siyuan, et al. (2018) Vibration characteristics analysis of the new pin-cycloid speed reducer. Journal of the Brazilian Society of Mechanical Sciences and Engineering, 40(2), 1- 17.

6. CHEN Chao, CHEN Jiabin.(2015) Efficiency analysis of two degrees of freedom epicyclic gear transmission and experimental validation. Mechanism and Machine Theory, 87, 115-130.

7. Zhang Yuefei, Li Yong. (2016) Dynamic load hysteresis performance test system of CORT precision reducer. Mechanical Design and Research, 32(05), 68-71. 